\title{
The fatty acid composition of muscles and fat depots of ostriches as influenced by genotype
}

\author{
L.C. Hoffman ${ }^{1 \#}$, M.M. Brand ${ }^{1,2}$, S.W.P. Cloete ${ }^{1,3}$ \& M. Muller ${ }^{2}$ \\ ${ }^{1}$ University of Stellenbosch, Department of Animal Sciences, Private Bag X1, Matieland, 7602, South Africa \\ ${ }^{2}$ University of Stellenbosch, Department of Food Science, Private Bag X1, Matieland, 7602, South Africa \\ ${ }^{3}$ Institute for Animal Production: Elsenburg, Private Bag XI, Elsenburg, 7607, South Africa
}

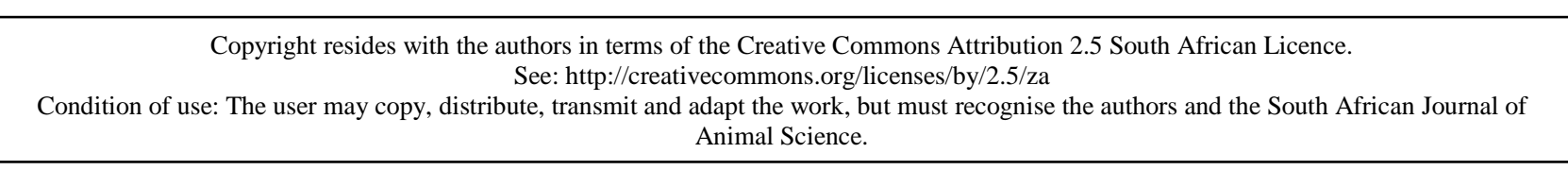

\begin{abstract}
Three genotypes of ostrich (South African Black, Zimbabwean Blue Necks and crosses between Zimbabwean Blue Neck males x South African Black females) were used to investigate the influence of genotype on the fatty acid composition of the musculus gastrocnemius and musculus iliofibularis, and abdominal and breast fat depots. Total saturated fatty acids in both the $m$. gastrocnemius and $m$. iliofibularis were higher in crosses (36.4\% and 35.9\%) than in South African Black ostriches (32.9\% and 30.4\%), whereas mono-unsaturated fatty acids were highest in South African Black ostriches. Total polyunsaturated fatty acids in the $m$. gastrocnemius were lowest in South African Black ostriches (26.5\%) compared with the Zimbabwean Blue Necks (33.2\%). Neither the ratio of polyunsaturated to saturated (0.73 to 0.99) nor $n-6$ to $n$-3 (1.6 to 2.2) fatty acids were influenced by genotype, although the ratio of polyunsaturated to saturated fatty acids in Zimbabwean Blue Necks was higher in the $m$. gastrocnemius $(0.99)$ than in the $m$. iliofibularis (0.73). Differences in individual fatty acids were prominent in the breast fat, and total saturated and monounsaturated fatty acids differed between genotypes in both breast and abdominal fat. Only the ratio of polyunsaturated to saturated fatty acids in the fat depots differed between South African Black (0.48) and Zimbabwean Blue Neck ostriches (0.42). The latter ratio was higher in the breast (0.50) than abdominal (0.42) fat in Zimbabwean Blue Necks. These results indicate that crossbreeding of different genotypes of ostriches reared under similar conditions does influence the overall fatty acid profiles of meat and fat.
\end{abstract}

Keywords: Struthio camelus, SFA, MUFA, PUFA, crossbreeding

" Corresponding author: lch@sun.ac.za

\section{Introduction}

Meat is seen as a major source of fat, especially saturated fatty acids, and it can contribute to various cardiovascular diseases and cancer. An augmented intake of saturated fatty acids increases the plasma level of low-density lipoprotein, while it is decreased by the intake of polyunsaturated and mono-unsaturated fatty acids (Sinclair et al., 1982; Mattson \& Grundy, 1985; Stipanuk, 2000).

Ostrich meat contains a relatively high concentration of polyunsaturated fatty acids (Sales, 1998; Girolami et al., 2003). The influences on fatty acid composition of muscle (Sales, 1998), genotype (Horbañczuk et al., 1998), animal age (Hoffman \& Fisher, 2001; Girolami et al., 2003) and feeding (Lanza et al., 2004; Hoffman et al., 2005) have been evaluated in ostrich meat. As opposed to what happens in broilers, extra-muscular fat forms a layer in the abdominal cavity and breast (sternum) of ostriches (Sales, 1999). However, there is a dearth of information about the fatty acid composition of the various ostrich fat depots. Ostrich fat depots are used in the meat processing trade as a fat source in processed products or sold locally where they are used in cooking as a source of lard.

Crossbreeding of genotypes of ostriches is practised to obtain a higher slaughter weight and faster growth rate, as well as direct heterosis (Engelbrecht et al., 2008). Information was presented that crossbreeding of South African Black (Struthio camelus var. domesticus) and Zimbabwean Blue Neck 
(Struthio camelus australis) ostriches presented higher absolute carcass and muscle weights than pure South African Black ostriches (Hoffman et al., 2007) without adversely influencing quality (lipid, protein, collagen, myoglobin, cholesterol, and minerals) of meat (Hoffman et al., 2008). It is known that genotype affects the concentration of fatty acids in meat animals and their adipose tissue (Boylan et al., 1976; Kemp et al., 1981; Sañudo et al., 1998). This investigation was thus undertaken to determine the effect of crossbreeding Zimbabwean Blue Neck and South African Black ostriches on the fatty acid composition of the musculus gastrocnemius, and musculus iliofibularis, as well as abdominal and breast fat depots, thereby providing the ostrich industry with scientific information about the effects of crossbreeding on the fatty acid profile of ostrich meat and fat.

\section{Materials and Methods}

Samples of $m$. gastrocnemius and $m$. iliofibularis muscles, and abdominal and breast fat depots of five South African Black ostriches $(\approx 85 \mathrm{~kg}$ live weight), two Zimbabwean Blue Necks $((\approx 101 \mathrm{~kg}$ live weight), and nine Zimbabwean Blue Neck male $x$ South African Black female crosses $(\approx 97 \mathrm{~kg}$ live weight) were evaluated for fatty acid composition. The $m$. gastrocnemius (big drum muscle) and $m$. iliofibularis (fan fillet muscle) were used for this analysis, as these two muscles are the largest (and most important), and are commonly available in the market (Mellett, 1992). It is conceded that the numbers of birds used were small. However, as pure Zimbabwean Blue Neck chicks exhibit a very poor survival (Engelbrecht et al., 2008), the experiment could only draw on the animal resources that were available in the same contemporary group. These birds were raised under the same conditions at Oudtshoorn Research Farm, near Oudtshoorn, South Africa. All birds received the same ad libitum diet (10.5 MJ energy, $160 \mathrm{~g}$ protein/kg dry matter) and had free access to drinking water during the entire growing-out phase. They were slaughtered commercially at 14 months of age, and muscles from the leg ( $m$. gastrocnemius and $m$. iliofibularis) and fat (breast and abdominal) were collected, as described by Hoffman et al. (2007). Fat samples were not homogenised or thawed in order to prevent oxidation of the fatty acids. When slaughtered, the fat concentrations of these muscles ranged from $22 \mathrm{~g} / \mathrm{kg} D M$ to $27 \mathrm{~g} / \mathrm{kg}$ DM for the $m$. iliofibularis and from $16 \mathrm{~g} / \mathrm{kg} \mathrm{DM}$ to $24 \mathrm{~g} / \mathrm{kg}$ DM for the $m$. gastrocnemius, but no conclusive significant differences could be demonstrated between genotypes (Brand, 2006).

The fatty acid concentration of the muscle and fat depots was determined using the method described by Tichelaar et al. (1998). Using thawed meat samples and frozen fat samples, the lipid in a $2 \mathrm{~g}$ sample of meat and $1 \mathrm{~g}$ sample of fat was extracted with chloroform/methanol (2:1; v/v) according to a modified method of Folch et al. (1957). All the extraction solvents contained 0.01\% butylated hydroxytoluene (BHT) as an antioxidant. A polytron mixer (Kinematica, type PT 10-35, Switzerland) was used to homogenize the sample within the extraction solvent. A sub-sample of the extracted lipids was transmethylated for $2 \mathrm{~h}$ at 70 ${ }^{\circ} \mathrm{C}$ using methanol/sulphuric acid (19:1; v/v). After cooling, the resulting fatty acid methyl esters (FAME) were extracted with water and hexane. The FAME were purified by using TLC Silica gel 60 plates (Merck, Cat no. 1.05721.0001) and analysed by GLC (Thermo Finnigan Focus GC equipped with flame ionisation detection), using $60 \mathrm{~m}$ BPX70 capillary columns of $0.25 \mathrm{~mm}$ internal diameter, $0.25 \mu \mathrm{m}$ film (SGE, Australia). Gas flow rates were hydrogen, $25 \mathrm{~mL} / \mathrm{min}$; and hydrogen carrier gas $2-4 \mathrm{~mL} / \mathrm{min}$. Temperature programming was linear at $4{ }^{\circ} \mathrm{C} / \mathrm{min}$, with an initial temperature of $140{ }^{\circ} \mathrm{C}$, a final temperature of $240{ }^{\circ} \mathrm{C}$, an injector temperature of $220^{\circ} \mathrm{C}$ and a detector temperature of $260{ }^{\circ} \mathrm{C}$. The FAME in the total lipids was identified by comparison of the retention times with those of a standard FAME mixture (Supelco® 37 Component FAME Mix, Catalogue no. 18919-1AMP, Lot no. LB-16064. Sigma Aldrich Inc. North Harrison Road, Bellefonte, PA 16823-0048, USA).

Regarding the fatty acid analysis of the meat and the breast fat, a two-factor factorial experiment was performed in a randomised block design with 16 block replications (carcasses). The factors were three genotypes (Black x Black, Blue x Black and Blue x Blue) and two muscles ( $m$. gastrocnemius and $m$. iliofibularis) for the meat samples, whereas two types of fat (breast fat and abdominal fat) were used to analyse the data of the fat samples. However, only 14 block replications (carcasses) were used in order to analyse the data of the abdominal fat samples. An experimental unit consisted of a single carcass. The variables were recorded as interval data and subjected to an analysis of variance (ANOVA) using SAS Version 9 Statistical Software (SAS, 1999). Regarding all the statistical analyses, the ANOVA was performed on the full model with factors and interactions included. The Shapiro-Wilk test was performed to test for non-normality (Shapiro \& Wilk, 1965). Student's t-least significant difference (LSD) was calculated 
at the 5\% significance level to compare treatment means (Ott, 1998). Results were defined as being not significant at a level of $P>0.05$ and significant at a level of $P \leq 0.05$. In a few cases, deviations from normality were the result of outliers, which were removed before the final analysis. Where there was still significant evidence of non-normality, this could be ascribed to kurtosis rather than skewness. Interpretation of results was thus continued as specified by Glass et al. (1972). Correlations were estimated using the Pearson product moment correlation coefficient.

\section{Results and Discussion}

The fatty acid composition (\% of total fatty acids present) of the $m$. gastrocnemius and $m$. iliofibularis from different genotypes of ostrich is presented in Table 1.

Although some of the saturated fatty acids (SFA) differed statistically between breeds, those that were present in concentrations lower than 1\% (C10:0, C12:0, C14:0, C20:0 and C22:0) will not be discussed. The saturated fatty acid, palmitic acid (C16:0), was found to be higher $(P<0.05)$ in the $m$. iliofibularis of the Zimbabwean Blue Necks than in that of South African Black ostriches. Total saturated fatty acids were lower $(P<0.05)$ in both the $m$. gastrocnemius and $m$. iliofibularis in crosses than in South African Black ostriches, with no differences $(P<0.05)$ being found in meat between crosses and Zimbabwean Blue Necks.

Similar to the SFA, the MUFA present $<1 \%$ will not be discussed. The $n-9$ mono-unsaturated fatty acids nervonic acid $(\mathrm{C} 24: 1 n-9)$ in the $m$. gastrocnemius, was higher $(P<0.05)$ in Zimbabwean Blue Necks than South African Black ostriches. However, total mono-unsaturated fatty (MUFA) acids were higher ( $P$ $<0.05$ ) in South African Black ostriches than either Zimbabwean Blue Necks or crosses in both the m. gastrocnemius and $m$. iliofibularis.

Eicosadienoic acid (C20:2), dihomo- $\gamma$-linolenic (C20:3n-6), arachidonic acid (C20:4n-6) and docosapentaenoic acid $(\mathrm{C} 22: 5-3)$ were higher $(P<0.05)$ in Zimbabwean Blue Necks than South African Black ostriches in the $m$. gastrocnemius, with C20:3n-6 in crosses similar $(P>0.05)$ to Zimbabwean Blue Necks. C20:3n-6 fatty acids in the m. iliofibularis were lowest $(P<0.05)$ in South African Black ostriches. Total polyunsaturated fatty acid (PUFA) content in the $m$. gastrocnemius of crosses was similar $(P>0.05)$ to the content in Zimbabwean Blue Necks and South African Black ostriches, with differences $(P<0.05)$ being found between the latter two genotypes, whereas no differences $(P>0.05)$ were detected in the m. iliofibularis.

The fatty acids, C20:2, C20:4n-6 and C22:5n-3, and desirable fatty acids (C18:0 + total unsaturated fatty acids) were higher $(P<0.05)$ in the $m$. gastrocnemius than the $m$. iliofibularis in Zimbabwean Blue Necks, but not $(P>0.05)$ in the other genotypes. No differences $(P>0.05)$ in the ratios of polyunsaturated to saturated or $n-6$ to $n-3$ fatty acids were detected in either genotype or muscle (Table 3 ). According to Girolami et al. (2003) and Lanza et al. (2004), no differences occurred in the ratio of polyunsaturated to saturated fatty acids in the $m$. gastrocnemius and $m$. iliofibularis in Zimbabwean Blue Necks and crosses between Zimbabwean Blue Neck and South African Black. However, Sales (1998) found this ratio was lowest in the m. iliofibularis in South African Black ostriches.

Whereas no differences have been reported in the ratio of $n-6$ to $n-3$ fatty acids in meat from Kenyan Red Neck (Struthio camelus massaicus) and Zimbabwean Blue Neck ostriches (Horbañczuk et al., 1998), this ratio was found to be higher in the $m$. gastrocnemius than in the m. iliofibularis in Zimbabwean Blue Neck and South African Black crosses (Lanza et al., 2004). However, Sales (1998) and Girolami et al. (2003) found no differences in the $n-6$ to $n-3$ ratio between the $m$. gastrocnemius and $m$. iliofibularis in South African Black ostriches and Zimbabwean Blue Necks, respectively. The ratio of $n-6$ to $n-3$ fatty acids reported in the literature (Sales et al., 1996; Horbañczuk et al., 1998; Sales, 1998; Hoffman \& Fisher, 2001, Girolami et al., 2003; Lanza et al., 2004; Hoffman et al., 2005) for ostrich meat varied from 2.50 to 9.65. Values of above 0.45 and below 4.0 for the ratios of polyunsaturated to saturated fatty acids and $n-6$ to $n-3$, respectively, are recommended by the British Department of Health (1994) in attempts to decrease cardiovascular diseases. In accordance with previous studies on the fatty acid composition of ostrich meat (Sales et al., 1996; Horbañczuk et al., 1998; Paleari et al., 1998; Sales, 1998; Hoffman \& Fisher, 2001; Girolami et al., 2003; Lanza et al., 2004; Hoffman et al., 2005), oleic acid (C18:1n-9c) was the dominant fatty acid found in both muscles (Table 1).

Individual fatty acids concentrations were relatively constant between genotypes in breast fat, with higher $(P<0.05)$ percentages found in Zimbabwean Blue Necks than South African Black ostriches for C16:0, stearic acid (C18:0), elaidic acid (C18:1n-9t) and C20:4n-6 (Table 2). 
Table 1 Means ( \pm s.d.) depicting the fatty acid composition (\% of total fatty acids present) of the musculus gastrocnemius and musculus iliofibularis from different ostrich genotypes

\begin{tabular}{|c|c|c|c|c|}
\hline $\begin{array}{l}\text { Fatty } \\
\text { acids }\end{array}$ & Muscles & $\begin{array}{l}\text { South African Black } \\
(\mathrm{n}=5)\end{array}$ & $\begin{array}{l}\text { Cross } \\
(\mathrm{n}=9)\end{array}$ & $\begin{array}{l}\text { Zimbabwean Blue Neck } \\
(\mathrm{n}=2)\end{array}$ \\
\hline \multirow{2}{*}{ C10:0 } & M. gastrocnemius & $0.00^{\mathrm{b}} \pm 0.000$ & $0.2^{\mathrm{a}} \pm 0.122$ & $0.16^{\mathrm{a}} \pm 0.120$ \\
\hline & M. iliofibularis & $0.02^{\mathrm{b}} \pm 0.035$ & $0.14^{\mathrm{b}} \pm 0.067$ & $0.13^{\mathrm{ab}} \pm 0.069$ \\
\hline \multirow{2}{*}{ C12:0 } & M. gastrocnemius & $0.09^{\mathrm{b}} \pm 0.110$ & $0.14^{\mathrm{ab}} \pm 0.048$ & $0.25^{\mathrm{a}} \pm 0.065$ \\
\hline & M. iliofibularis & $0.07 \pm 0.101$ & $0.15 \pm 0.075$ & $0.20 \pm 0.044$ \\
\hline \multirow{2}{*}{ C14:0 } & M. gastrocnemius & $0.51_{a} \pm 0.181$ & $0.38 \pm 0.093$ & $0.36 \pm 0.253$ \\
\hline & M. iliofibularis & $0.23_{b} \pm 0.188$ & $0.35 \pm 0.104$ & $0.38 \pm 0.288$ \\
\hline \multirow{2}{*}{ C16:0 } & M. gastrocnemius & $18.3 \pm 1.60$ & $17.3 \pm 2.45$ & $17.8 \pm 0.06$ \\
\hline & M. iliofibularis & $16.5^{\mathrm{b}} \pm 1.89$ & $19.2^{\mathrm{a}} \pm 3.19$ & $19.9^{\mathrm{a}} \pm 2.11$ \\
\hline \multirow{2}{*}{ C18:0 } & M. gastrocnemius & $11.2 \pm 2.28$ & $11.8 \pm 2.75$ & $10.3 \pm 1.38$ \\
\hline & M. iliofibularis & $11.0 \pm 1.04$ & $10.0 \pm 1.31$ & $10.6 \pm 2.03$ \\
\hline \multirow{2}{*}{ C20:0 } & M. gastrocnemius & $0.18 \pm 0.070$ & $0.21 \pm 0.105$ & $0.14 \pm 0.052$ \\
\hline & M. iliofibularis & $0.14 \pm 0.040$ & $0.56 \pm 0.746$ & $0.14 \pm 0.065$ \\
\hline \multirow{2}{*}{ C22:0 } & M. gastrocnemius & $0.17^{\mathrm{ab}} \pm 0.096$ & $0.27^{\mathrm{a}} \pm 0.173$ & $0.11^{\mathrm{b}} \pm 0.000$ \\
\hline & M. iliofibularis & $0.16 \pm 0.076$ & $0.13 \pm 0.070$ & $0.20 \pm 0.117$ \\
\hline \multirow{2}{*}{ C24:0 } & M. gastrocnemius & $1.10 \pm 0.476$ & $1.10 \pm 0.632$ & $1.13 \pm 0.118$ \\
\hline & M. iliofibularis & $0.98 \pm 0.223$ & $0.84 \pm 0.219$ & $1.01 \pm 1.144$ \\
\hline \multirow{2}{*}{ C14:1 } & M. gastrocnemius & $0.09 \pm 0.058$ & $0.15 \pm 0.092$ & $0.11 \pm 0.069$ \\
\hline & M. iliofibularis & $0.10 \pm 0.065$ & $0.18 \pm 0.229$ & $0.05 \pm 0.072$ \\
\hline \multirow{2}{*}{ C16:1n-7 } & M. gastrocnemius & $5.6 \pm 1.30$ & $4.5 \pm 1.17$ & $5.0 \pm 0.80$ \\
\hline & M. iliofibularis & $4.6 \pm 0.93$ & $5.2 \pm 1.15$ & $5.1 \pm 2.02$ \\
\hline \multirow{2}{*}{ C18:1n-9t } & M. gastrocnemius & $0.24 \pm 0.085$ & $0.55 \pm 0.688$ & $0.19 \pm 0.034$ \\
\hline & M. iliofibularis & $0.24 \pm 0.097$ & $0.57 \pm 0.452$ & $0.27 \pm 0.003$ \\
\hline \multirow{2}{*}{ C18:1n-9c } & M. gastrocnemius & $27.4 \pm 1.35$ & $26.5 \pm 2.97$ & $25.1 \pm 0.04$ \\
\hline & M. iliofibularis & $29.3^{\mathrm{a}} \pm 3.74$ & $26.7^{\mathrm{ab}} \pm 2.85$ & $26.4^{\mathrm{b}} \pm 2.02$ \\
\hline \multirow{2}{*}{ C20:1n-9 } & M. gastrocnemius & $0.26 \pm 0.046$ & $0.30 \pm 0.095$ & $0.25 \pm 0.010$ \\
\hline & M. iliofibularis & $0.29 \pm 0.032$ & $0.25 \pm 0.047$ & $0.28 \pm 0.039$ \\
\hline \multirow{2}{*}{ C22:1n-9 } & M. gastrocnemius & $0.08 \pm 0.089$ & $0.13 \pm 0.070$ & $0.14_{b} \pm 0.087$ \\
\hline & M. iliofibularis & $0.10^{\mathrm{b}} \pm 0.078$ & $0.08^{\mathrm{b}} \pm 0.045$ & $0.37^{\mathrm{a}}{ }_{\mathrm{a}} \pm 0.245$ \\
\hline \multirow{2}{*}{ C24:1n-9 } & M. gastrocnemius & $0.3^{\mathrm{b}} \pm 0.15$ & $0.6^{b} \pm 0.37$ & $2.3^{\mathrm{a}} \pm 0.53$ \\
\hline & M. iliofibularis & $1.0 \pm 1.37$ & $0.6 \pm 0.60$ & $1.5 \pm 0.33$ \\
\hline
\end{tabular}


Table 1 (continued) Means ( \pm s.d.) depicting the fatty acid composition (\% of total fatty acids present) of the musculus gastrocnemius and musculus iliofibularis from different ostrich genotypes

\begin{tabular}{|c|c|c|c|c|}
\hline $\begin{array}{l}\text { Fatty } \\
\text { acids }\end{array}$ & Muscles & $\begin{array}{l}\text { South African Black } \\
(\mathrm{n}=5)\end{array}$ & $\begin{array}{l}\text { Cross } \\
(\mathrm{n}=9)\end{array}$ & $\begin{array}{c}\text { Zimbabwean Blue Neck } \\
(\mathrm{n}=2)\end{array}$ \\
\hline \multirow{2}{*}{ C18:2n-6t } & M. gastrocnemius & $1.7 \pm 1.48$ & $1.1 \pm 1.41$ & $1.2 \pm 1.48$ \\
\hline & M. iliofibularis & $2.4 \pm 1.36$ & $1.2 \pm 1.09$ & $1.2 \pm 1.62$ \\
\hline \multirow{2}{*}{ C18:2n-6c } & M. gastrocnemius & $12.6 \pm 0.92$ & $13.1 \pm 1.17$ & $12.8 \pm 0.10$ \\
\hline & M. iliofibularis & $13.7 \pm 1.48$ & $13.5 \pm 0.99$ & $12.3 \pm 2.20$ \\
\hline \multirow{2}{*}{ C18:3n -6 } & M. gastrocnemius & $0.05 \pm 0.053$ & $0.52 \pm 0.491$ & $0.19 \pm 0.120$ \\
\hline & M. iliofibularis & $0.09 \pm 0.065$ & $0.65 \pm 0.911$ & $0.31 \pm 0.050$ \\
\hline \multirow{2}{*}{ C18:3n -3 } & M. gastrocnemius & $1.7 \pm 0.50$ & $1.5 \pm 0.40$ & $1.7 \pm 0.35$ \\
\hline & M. iliofibularis & $1.3 \pm 0.66$ & $1.6 \pm 0.83$ & $1.6 \pm 0.25$ \\
\hline \multirow{2}{*}{ C20:2 } & M. gastrocnemius & $0.2^{\mathrm{b}} \pm 0.04$ & $0.4^{\mathrm{b}} \pm 0.34$ & $1.1^{\mathrm{a}}{ }_{\mathrm{a}} \pm 1.17$ \\
\hline & M. iliofibularis & $0.3 \pm 0.10$ & $0.3 \pm 0.37$ & $0.2_{\mathrm{b}} \pm 0.05$ \\
\hline \multirow{2}{*}{ C20:3n-6 } & M. gastrocnemius & $0.6^{\mathrm{b}} \pm 0.08$ & $1.9^{\mathrm{a}} \pm 0.51$ & $2.1^{\mathrm{a}} \pm 0.09$ \\
\hline & M. iliofibularis & $0.7^{\mathrm{b}} \pm 0.33$ & $1.5^{\mathrm{a}} \pm 0.31$ & $2.0^{\mathrm{a}} \pm 0.20$ \\
\hline \multirow{2}{*}{ C20:3n-3 } & M. gastrocnemius & $7.2 \pm 2.68$ & $6.8 \pm 1.84$ & $8.9 \pm 0.73$ \\
\hline & M. iliofibularis & $6.5 \pm 2.38$ & $6.1 \pm 1.17$ & $6.9 \pm 3.35$ \\
\hline \multirow{2}{*}{ C20:4n-6 } & M. gastrocnemius & $0.2^{\mathrm{b}} \pm 0.08$ & $0.4^{\mathrm{b}} \pm 0.37$ & $1.1^{\mathrm{a}}{ }_{\mathrm{a}} \pm 0.00$ \\
\hline & M. iliofibularis & $0.2 \pm 0.08$ & $0.5 \pm 0.41$ & $0.5_{b} \pm 0.42$ \\
\hline \multirow{2}{*}{ C20:5n-3 } & M. gastrocnemius & $0.55 \pm 0.172$ & $0.55 \pm 0.285$ & $0.70 \pm 0.071$ \\
\hline & M. iliofibularis & $0.45 \pm 0.181$ & $0.52 \pm 0.256$ & $0.46 \pm 0.090$ \\
\hline \multirow{2}{*}{ C22:2 } & M. gastrocnemius & $0.3 \pm 0.32$ & $2.6 \pm 4.16$ & $0.6 \pm 0.81$ \\
\hline & M. iliofibularis & $0.4 \pm 0.29$ & $2.3 \pm 2.83$ & $0.3 \pm 0.19$ \\
\hline \multirow{2}{*}{ C22:5n-3 } & M. gastrocnemius & $0.8^{\mathrm{b}} \pm 0.50$ & $0.6^{\mathrm{b}} \pm 0.57$ & $1.8^{\mathrm{a}}{ }_{\mathrm{a}} \pm 0.01$ \\
\hline & M. iliofibularis & $0.9 \pm 0.52$ & $0.9 \pm 0.38$ & $0.3_{\mathrm{b}} \pm 0.08$ \\
\hline \multirow{2}{*}{ C22:6n-3 } & M. gastrocnemius & $0.6 \pm 0.22$ & $0.8 \pm 0.49$ & $1.1 \pm 0.11$ \\
\hline & M. iliofibularis & $0.7 \pm 0.38$ & $0.7 \pm 0.38$ & $1.1 \pm 0.91$ \\
\hline \multirow{2}{*}{ SFA } & M. gastrocnemius & $32.9^{\mathrm{b}} \pm 2.74$ & $36.4^{\mathrm{a}} \pm 1.67$ & $33.7^{\mathrm{ab}} \pm 1.24$ \\
\hline & M. iliofibularis & $30.4^{\mathrm{b}} \pm 1.46$ & $35.9^{\mathrm{a}} \pm 2.75$ & $38.6^{\mathrm{a}}{ }_{\mathrm{a}} \pm 6.16$ \\
\hline \multirow{2}{*}{ MUFA } & M. gastrocnemius & $40.6^{\mathrm{a}} \pm 4.22$ & $33.2^{\mathrm{b}} \pm 3.96$ & $33.2^{\mathrm{b}} \pm 0.31$ \\
\hline & M. iliofibularis & $41.9^{\mathrm{a}} \pm 3.07$ & $34.2^{b} \pm 3.76$ & $34.2^{\mathrm{b}} \pm 0.02$ \\
\hline \multirow{2}{*}{ PUFA } & M. gastrocnemius & $26.5^{\mathrm{b}} \pm 5.34$ & $30.4^{\mathrm{ab}} \pm 4.67$ & $33.2^{\mathrm{a}} \pm 0.93$ \\
\hline & M. iliofibularis & $27.7 \pm 2.82$ & $29.9 \pm 4.51$ & $27.2 \pm 6.18$ \\
\hline \multirow{2}{*}{ DFA } & M. gastrocnemius & $78.3 \pm 1.25$ & $75.4 \pm 2.43$ & $76.6_{a} \pm 2.62$ \\
\hline & M. iliofibularis & $80.6^{\mathrm{a}} \pm 1.60$ & $74.1^{\mathrm{b}} \pm 2.97$ & $72.0^{\mathrm{b}} \mathrm{b} \pm 8.19$ \\
\hline
\end{tabular}

${ }^{\mathrm{a}, \mathrm{b}}$ Row means with common superscripts do not differ $(P<0.05)$.

a,b Column means within each fatty acid with common subscripts do not differ $(P<0.05)$.

SFA - saturated fatty acids; MUFA - mono-unsaturated fatty acids; PUFA - polyunsaturated fatty acids;

DFA - desirable fatty acids (C18:0 + total unsaturated fatty acids). 
Table 2 Means ( \pm s.d.) depicting the fatty acid composition (\% of total fatty acids present) of the breast and abdominal fat from different ostrich genotypes

\begin{tabular}{|c|c|c|c|c|}
\hline $\begin{array}{l}\text { Fatty } \\
\text { acids }\end{array}$ & Fat depot & $\begin{array}{l}\text { South African Black } \\
\qquad(\mathrm{n}=5)\end{array}$ & $\begin{array}{l}\text { Cross } \\
(\mathrm{n}=9)\end{array}$ & $\begin{array}{c}\text { Zimbabwean Blue } \\
\text { Neck } \\
(\mathrm{n}=2) \\
\end{array}$ \\
\hline \multirow{2}{*}{ C10:0 } & Breast & $0.02 \pm 0.016$ & $0.01 \pm 0.017$ & $0.01 \pm 0.000$ \\
\hline & Abdominal & $0.02 \pm 0.014$ & $0.01 \pm 0.007$ & $0.04 \pm 0.000$ \\
\hline \multirow{2}{*}{ C12:0 } & Breast & $0.08 \pm 0.038$ & $0.08 \pm 0.022$ & $0.09 \pm 0.057$ \\
\hline & Abdominal & $0.06^{\mathrm{b}} \pm 0.042$ & $0.09^{\mathrm{ab}} \pm 0.028$ & $0.13^{\mathrm{a}} \pm 0.000$ \\
\hline \multirow{2}{*}{ C14:0 } & Breast & $1.12 \pm 0.141$ & $1.06 \pm 0.131$ & $1.24 \pm 0.036$ \\
\hline & Abdominal & $1.09^{\mathrm{b}} \pm 0.075$ & $1.11^{\mathrm{b}} \pm 0.111$ & $1.37^{\mathrm{a}} \pm 0.000$ \\
\hline \multirow{2}{*}{ C16:0 } & Breast & $29.6^{\mathrm{b}} \pm 0.57$ & $30.2^{\mathrm{ab}} \pm 1.31$ & $30.8^{\mathrm{a}} \pm 1.28$ \\
\hline & Abdominal & $30.3 \pm 0.57$ & $30.4 \pm 0.79$ & $30.6 \pm 0.00$ \\
\hline \multirow{2}{*}{ C18:0 } & Breast & $5.0^{\mathrm{b}}{ }_{\mathrm{b}} \pm 0.42$ & $4.9^{\mathrm{b}}{ }_{\mathrm{b}} \pm 0.50$ & $5.7^{\mathrm{a}}{ }_{\mathrm{a}} \pm 1.18$ \\
\hline & Abdominal & $5.6^{\mathrm{a}}{ }_{\mathrm{a}}^{\mathrm{a}} \pm 0.27$ & $5.5^{\mathrm{a}}{ }_{\mathrm{a}} \pm 0.47$ & $4.8^{\mathrm{b}}{ }_{\mathrm{b}}^{\mathrm{a}} \pm 0.00$ \\
\hline \multirow[b]{2}{*}{ C20:0 } & Breast & $0.05 \pm 0.017$ & $0.038 \pm 0.011$ & $0.05 \pm 0.010$ \\
\hline & Abdominal & $0.05^{\mathrm{ab}} \pm 0.007$ & $0.045^{\mathrm{b}} \pm 0.001$ & $0.06^{\mathrm{a}} \pm 0.000$ \\
\hline \multirow{2}{*}{ C22:0 } & Breast & $0.02 \pm 0.005$ & $0.11 \pm 0.261$ & $0.01 \pm 0.002$ \\
\hline & Abdominal & $0.02 \pm 0.007$ & $0.01 \pm 0.010$ & $0.01 \pm 0.000$ \\
\hline \multirow{2}{*}{ C24:0 } & Breast & $0.40 \pm 0.295$ & $0.42 \pm 0.196$ & $0.08_{b} \pm 0.088$ \\
\hline & Abdominal & $0.33^{\mathrm{b}} \pm 0.288$ & $0.28^{\mathrm{b}} \pm 0.197$ & $2.84^{\mathrm{a}}{ }_{\mathrm{a}} \pm 0.000$ \\
\hline \multirow{2}{*}{ C14:1 } & Breast & $0.16 \pm 0.034$ & $0.13 \pm 0.044$ & $0.16 \pm 0.029$ \\
\hline & Abdominal & $0.14^{\mathrm{b}} \pm 0.022$ & $0.15^{\mathrm{b}} \pm 0.029$ & $0.21^{\mathrm{a}} \pm 0.000$ \\
\hline \multirow{2}{*}{ C16:1n-7 } & Breast & $9.7 \pm 1.02$ & $9.6 \pm 0.89$ & $9.5 \pm 2.38$ \\
\hline & Abdominal & $9.2^{\mathrm{b}} \pm 1.17$ & $9.7^{\mathrm{ab}} \pm 0.88$ & $10.5^{\mathrm{a}} \pm 0.00$ \\
\hline \multirow{2}{*}{ C18:1n-9t } & Breast & $0.10^{\mathrm{b}}{ }_{\mathrm{b}} \pm 0.103$ & $0.19^{\mathrm{ab}} \pm 0.066$ & $0.21^{\mathrm{a}}{ }_{\mathrm{a}} \pm 0.027$ \\
\hline & Abdominal & $0.22^{\mathrm{a}}{ }_{\mathrm{a}} \pm 0.013$ & $0.22^{\mathrm{a}} \pm 0.021$ & $0.01^{b}{ }_{b} \pm 0.000$ \\
\hline \multirow{2}{*}{ C18:1n-9c } & Breast & $33.3 \pm 1.34$ & $33.2 \pm 1.29$ & $32.1_{a} \pm 0.59$ \\
\hline & Abdominal & $33.7^{\mathrm{a}} \pm 0.60$ & $32.5^{\mathrm{a}} \pm 0.98$ & $30.1_{b}^{b} \pm 0.00$ \\
\hline \multirow{2}{*}{ C20:1n-9 } & Breast & $0.23 \pm 0.040$ & $0.21 \pm 0.028$ & $0.21 \pm 0.061$ \\
\hline & Abdominal & $0.24^{\mathrm{a}} \pm 0.026$ & $0.22^{\mathrm{ab}} \pm 0.028$ & $0.21^{b} \pm 0.000$ \\
\hline \multirow{2}{*}{ C22:1n-9 } & Breast & $0.01 \pm 0.005$ & $0.02 \pm 0.014$ & $0.01 \pm 0.002$ \\
\hline & Abdominal & $0.02 \pm 0.010$ & $0.02 \pm 0.004$ & $0.01 \pm 0.000$ \\
\hline \multirow{2}{*}{ C24:1n-9 } & Breast & $0.7 \pm 0.73$ & $0.5 \pm 0.44$ & $0.3_{\mathrm{b}} \pm 0.33$ \\
\hline & Abdominal & $0.3^{\mathrm{b}} \pm 0.13$ & $0.3^{\mathrm{b}} \pm 0.24$ & $1.3^{\mathrm{a}}{ }_{\mathrm{a}} \pm 0.00$ \\
\hline
\end{tabular}


Table 2 (continued) Means ( \pm s.d.) depicting the fatty acid composition (\% of total fatty acids present) of the breast and abdominal fat from different ostrich genotypes

\begin{tabular}{|c|c|c|c|c|}
\hline $\begin{array}{l}\text { Fatty } \\
\text { acids }\end{array}$ & Fat depot & $\begin{array}{l}\text { South African Black } \\
\qquad(\mathrm{n}=5)\end{array}$ & $\begin{array}{l}\text { Cross } \\
(\mathrm{n}=9)\end{array}$ & $\begin{array}{c}\text { Zimbabwean Blue } \\
\text { Neck } \\
(\mathrm{n}=2) \\
\end{array}$ \\
\hline $\mathrm{C} 18: 2 n-6 \mathrm{t}$ & $\begin{array}{l}\text { Breast } \\
\text { Abdominal }\end{array}$ & $\begin{array}{r}0.02 \pm 0.010 \\
0.04^{\mathrm{a}} \pm 0.026\end{array}$ & $\begin{array}{r}0.02 \pm 0.003 \\
0.02^{\mathrm{ab}} \pm 0.007\end{array}$ & $\begin{array}{r}0.03 \pm 0.011 \\
0.01^{\mathrm{b}} \pm 0.000\end{array}$ \\
\hline C18:2n-6c & $\begin{array}{l}\text { Breast } \\
\text { Abdominal }\end{array}$ & $\begin{array}{r}14.0 \pm 0.84 \\
14.3^{\mathrm{a}} \pm 1.28\end{array}$ & $\begin{array}{r}14.1 \pm 1.30 \\
14.2^{\mathrm{a}} \pm 0.85\end{array}$ & $\begin{array}{r}14.3_{\mathrm{a}} \pm 2.43 \\
12.4^{\mathrm{b}}{ }_{\mathrm{b}} \pm 0.00\end{array}$ \\
\hline C18:3n -6 & $\begin{array}{l}\text { Breast } \\
\text { Abdominal }\end{array}$ & $\begin{array}{l}0.10 \pm 0.053 \\
0.12 \pm 0.025\end{array}$ & $\begin{array}{l}0.10 \pm 0.039 \\
0.09 \pm 0.023\end{array}$ & $\begin{array}{l}0.10 \pm 0.011 \\
0.10 \pm 0.000\end{array}$ \\
\hline C18:3n -3 & $\begin{array}{l}\text { Breast } \\
\text { Abdominal }\end{array}$ & $\begin{array}{l}3.6 \pm 0.40 \\
2.8 \pm 1.54\end{array}$ & $\begin{array}{l}3.4 \pm 0.93 \\
3.5 \pm 0.51\end{array}$ & $\begin{array}{l}3.6 \pm 0.28 \\
3.2 \pm 0.00\end{array}$ \\
\hline C20:2 & $\begin{array}{l}\text { Breast } \\
\text { Abdominal }\end{array}$ & $\begin{array}{l}0.11 \pm 0.075 \\
0.12 \pm 0.030\end{array}$ & $\begin{array}{l}0.20 \pm 0.250 \\
0.13 \pm 0.069\end{array}$ & $\begin{array}{l}0.10 \pm 0.007 \\
0.07 \pm 0.000\end{array}$ \\
\hline C20:3n-6 & $\begin{array}{l}\text { Breast } \\
\text { Abdominal }\end{array}$ & $\begin{array}{l}0.07 \pm 0.062 \\
0.09 \pm 0.044\end{array}$ & $\begin{array}{l}0.18 \pm 0.216 \\
0.08 \pm 0.030\end{array}$ & $\begin{array}{l}0.13 \pm 0.013 \\
0.08 \pm 0.000\end{array}$ \\
\hline C20:3n-3 & $\begin{array}{l}\text { Breast } \\
\text { Abdominal }\end{array}$ & $\begin{array}{r}0.12^{\mathrm{b}} \pm 0.112 \\
0.18 \pm 0.081\end{array}$ & $\begin{array}{r}0.20^{\mathrm{ab}} \pm 0.116 \\
0.16 \pm 0.078\end{array}$ & $\begin{array}{r}0.26^{\mathrm{a}}{ }_{\mathrm{a}} \pm 0.119 \\
0.05_{\mathrm{b}} \pm 0.000\end{array}$ \\
\hline C20:4n-6 & $\begin{array}{l}\text { Breast } \\
\text { Abdominal }\end{array}$ & $\begin{array}{r}0.17^{\mathrm{a}} \pm 0.145 \\
0.09 \pm 0.120\end{array}$ & $\begin{array}{r}0.05^{\mathrm{b}} \pm 0.025 \\
0.05 \pm 0.032\end{array}$ & $\begin{array}{r}0.02^{\mathrm{b}} \pm 0.017 \\
0.01 \pm 0.000\end{array}$ \\
\hline C20:5n-3 & $\begin{array}{l}\text { Breast } \\
\text { Abdominal }\end{array}$ & $\begin{array}{l}0.03 \pm 0.006 \\
0.03 \pm 0.014\end{array}$ & $\begin{array}{l}0.04 \pm 0.018 \\
0.03 \pm 0.011\end{array}$ & $\begin{array}{l}0.05_{\mathrm{a}} \pm 0.015 \\
0.01_{b} \pm 0.000\end{array}$ \\
\hline C22:2 & $\begin{array}{l}\text { Breast } \\
\text { Abdominal }\end{array}$ & $\begin{array}{l}0.2 \pm 0.43 \\
0.2 \pm 0.36\end{array}$ & $\begin{array}{l}0.1 \pm 0.33 \\
0.2 \pm 0.36\end{array}$ & $\begin{array}{l}0.0 \pm 0.00 \\
0.1 \pm 0.00\end{array}$ \\
\hline C22:5n-3 & $\begin{array}{l}\text { Breast } \\
\text { Abdominal }\end{array}$ & $\begin{array}{r}0.3 \pm 0.34 \\
0.1^{b} \pm 0.08\end{array}$ & $\begin{array}{r}0.2 \pm 0.22 \\
0.2^{\mathrm{b}} \pm 0.40\end{array}$ & $\begin{array}{r}0.5 \pm 0.72 \\
0.8^{\mathrm{a}} \pm 0.00\end{array}$ \\
\hline C22:6n-3 & $\begin{array}{l}\text { Breast } \\
\text { Abdominal }\end{array}$ & $\begin{array}{l}0.1 \pm 0.03 \\
0.2 \pm 0.34\end{array}$ & $\begin{array}{l}0.2 \pm 0.36 \\
0.2 \pm 0.49\end{array}$ & $\begin{array}{l}0.1 \pm 0.10 \\
0.2 \pm 0.00\end{array}$ \\
\hline SFA & $\begin{array}{l}\text { Breast } \\
\text { Abdominal }\end{array}$ & $\begin{array}{l}37.0^{\mathrm{b}} \pm 0.68 \\
37.9^{\mathrm{b}} \pm 0.68\end{array}$ & $\begin{array}{r}37.3^{\mathrm{ab}} \pm 1.70 \\
38.1^{\mathrm{b}} \pm 1.17\end{array}$ & $\begin{array}{l}38.3^{\mathrm{a}}{ }_{\mathrm{b}} \pm 0.06 \\
40.7^{\mathrm{a}}{ }_{\mathrm{a}} \pm 0.00\end{array}$ \\
\hline MUFA & $\begin{array}{l}\text { Breast } \\
\text { Abdominal }\end{array}$ & $\begin{array}{l}44.3^{\mathrm{a}} \pm 1.00 \\
43.9^{\mathrm{a}} \pm 0.70\end{array}$ & $\begin{array}{r}43.9^{\mathrm{a}} \pm 1.55 \\
43.1^{\mathrm{ab}} \pm 1.16\end{array}$ & $\begin{array}{l}42.4^{\mathrm{b}} \pm 2.06 \\
42.4^{\mathrm{b}} \pm 0.00\end{array}$ \\
\hline PUFA & $\begin{array}{l}\text { Breast } \\
\text { Abdominal }\end{array}$ & $\begin{array}{r}18.7 \pm 0.94 \\
18.2^{\text {ab }} \pm 1.00\end{array}$ & $\begin{array}{r}18.8 \pm 1.87 \\
18.8^{\mathrm{a}} \pm 1.18\end{array}$ & $\begin{array}{r}19.2_{\mathrm{a}} \pm 1.99 \\
16.9^{\mathrm{b}}{ }_{\mathrm{b}} \pm 0.00\end{array}$ \\
\hline DFA & $\begin{array}{l}\text { Breast } \\
\text { Abdominal }\end{array}$ & $\begin{array}{r}68.0 \pm 0.53 \\
67.7^{\mathrm{a}} \pm 0.53\end{array}$ & $\begin{array}{r}67.6 \pm 1.38 \\
67.4^{\mathrm{a}} \pm 1.05\end{array}$ & $\begin{array}{r}67.3_{\mathrm{a}} \pm 1.12 \\
64.1^{\mathrm{b}}{ }_{\mathrm{b}} \pm 0.00\end{array}$ \\
\hline
\end{tabular}

\footnotetext{
${ }^{\mathrm{a}, \mathrm{b}}$ Row means with common superscripts do not differ $(P<0.05)$.

a,b Column means within each fatty acid with common subscripts do not differ $(P<0.05)$.

SFA - saturated fatty acids; MUFA - mono-unsaturated fatty acids; PUFA - polyunsaturated fatty acids;

DFA - desirable fatty acids (C18:0 + total unsaturated fatty acids).
} 
However, differences $(P<0.05)$ were prominent in several individual fatty acids between genotypes for abdominal fat, although no differences $(P>0.05)$ were found between total mono-unsaturated, polyunsaturated and desirable fatty acids, or in the ratio between polyunsaturated and saturated or $n-6$ and $n$-3 fatty acids (Table 3). In both breast and abdominal fat the total SFA concentration was lower $(P<0.05)$ in South African Black than in Zimbabwean Blue Neck ostriches, with total MUFA being higher $(P<0.05)$, possibly owing to differences $(P<0.05)$ in $\mathrm{C} 18: 1 n-9$ concentrations.

Where different $(P<0.05)$, individual fatty acid percentages were higher in the breast than in abdominal fat in the Zimbabwean Blue Neck genotype, as illustrated by a higher $(P<0.05)$ concentration of polyunsaturated and desirable fatty acids and in the ratio of polyunsaturated to saturated fatty acids (Table 2). As with the ostrich meat (Table 1), C18:1n-9c was the most abundant fatty acid in ostrich fat, followed by C16:0. The polyunsaturated fatty acid linoleic acid, (C18:2n-6c), was present in the highest concentration. In agreement with these results, Gunstone \& Russell (1954) reported the linolenic acid (C18:3) in ostrich fat as $3.8 \%$. Total saturated fatty acid concentrations of $29 \%$ to $47 \%$ have been reported for ostrich fat (Gunstone \& Russell, 1954; Joubert, 2003), whereas the polyunsaturated to saturated and $n-6$ to $n-3$ ratios in emu fat (abdominal and back) have been found to be $6.36 \%$ and $0.43 \%$, respectively (Wang et al., 2000). According to Hoffman et al. (2005) as with other monogastric species, the fatty acid composition of ostrich fat can be altered by manipulating the dietary fatty acids. The influence of diet on fat composition is illustrated in emu oil, in which contents of $\alpha$-linolenic acid (C18:3n-3) can vary from almost $0 \%$ in many farmed birds to up to $20 \%$ in some feral birds (Whitehouse et al., 1998).

\section{Conclusions}

The ostrich production industry needs scientific guidelines for crossbreeding ostriches. The effect of ostrich genotype, including South African Black (S. camelus var. domesticus), Zimbabwean Blue (S. camelus australis) and the genotype resulting from crossbreeding between these two genotypes, on the fatty acid composition of their meat and fat depots, was investigated. Genotype influenced the fatty acid composition of the muscles, as well as the fat depots. The results as a whole indicate that the pure Black genotype has a more positive unsaturated fatty acid profile in the $m$. iliofibularis and $m$. gastrocnemius and fat depots, while the fat depots of the Blue $x$ Black genotype seem to have a more positive unsaturated fatty acid profile. However, the ratios of polyunsaturated to saturated and $n-6$ to $n-3$ fatty acids are similar among South African Black ostriches, Zimbabwean Blue Necks, and crosses between these genotypes. Within genotype, there were few differences in individual fatty acids between the two muscles evaluated.

Table 3 Means ( \pm s.d.) depicting the ratios of polyunsaturated : saturated fatty acids and n-6:n-3 fatty acids ( \pm s.d.) in $\mathrm{mg} / \mathrm{g}$ sample of the musculus gastrocnemius and musculus iliofibularis and the breast and abdominal fat from different ostrich genotypes

\begin{tabular}{llrrr}
\hline $\begin{array}{l}\text { Fatty } \\
\text { acids }\end{array}$ & Muscles & $\begin{array}{c}\text { South African Black } \\
(\mathrm{n}=5)\end{array}$ & $\begin{array}{r}\text { Cross } \\
(\mathrm{n}=9)\end{array}$ & $\begin{array}{r}\text { Zimbabwean Blue Neck } \\
(\mathrm{n}=2)\end{array}$ \\
\hline & M. gastrocnemius & $0.81 \pm 0.200$ & $0.84 \pm 0.159$ & $0.99_{\mathrm{a}} \pm 0.064$ \\
P:S & M. iliofibularis & $0.91 \pm 0.105$ & $0.84 \pm 0.170$ & $0.73_{\mathrm{b}} \pm 0.276$ \\
& & & & \\
$n-6: n-3$ & M. gastrocnemius & $2.0 \pm 0.44$ & $2.1 \pm 0.48$ & $2.6 \pm 0.11$ \\
& M. iliofibularis & $2.2 \pm 0.61$ & $2.2 \pm 0.63$ & $2.2 \pm 0.91$ \\
P:S & Breast & $0.50 \pm 0.029$ & $0.51 \pm 0.066$ & $0.50_{\mathrm{a}} \pm 0.051$ \\
& Abdominal & $0.48^{\mathrm{a}} \pm 0.033$ & $0.49^{\mathrm{a}} \pm 0.040$ & $0.42^{\mathrm{b}}{ }_{\mathrm{b}} \pm 0.000$ \\
$n-6: n-3$ & Breast & $3.6 \pm 0.46$ & $3.9 \pm 1.56$ & $3.3 \pm 0.82$ \\
& Abdominal & $4.5 \pm 1.73$ & $3.7 \pm 0.95$ & $3.0 \pm 0.00$ \\
\hline
\end{tabular}

P:S - polyunsaturated to saturated fatty acids.

${ }^{\mathrm{a}, \mathrm{b}}$ Row means with common superscripts do not differ $(P<0.05)$.

a,b Column means within each fatty acid ratio with common subscripts do not differ $(P<0.05)$. 
However, as the meat from all the ostrich genotypes investigated in this study can contribute towards a healthy lifestyle, it is postulated that the specific genotype differences in chemical composition encountered in this investigation will not affect human health negatively.

This study was constrained through low animal numbers, particularly in the Zimbabwean Blue Neck group. However, it seemed that the ratios of polyunsaturated to saturated and $n-6$ to $n-3$ fatty acids are similar between South African Black ostriches, Zimbabwean Blue Necks and the cross of the Zimbabwean Blue Neck male with the South African Black female. In the absence of other results on fatty acid composition in the muscles and fat depots of ostriches, these findings may serve as preliminary indications of a lack of breed differences in these species.

More studies on ostrich fat depots should be undertaken, since research on the fatty acid composition of the ostrich fat is limited. Horbaňczuk et al. (2004) noted that ostrich adipose tissue could be used to supplement human diets. According to Fezler (1995) and Sales (1999), oils from the emu and rhea are used in cosmetics and first-aid products, but the reasons for the effectiveness and uniqueness of the oils are still unknown. Considering that the ostrich forms part of the same order of birds, namely Ratites, ostrich oil should be investigated further to exploit possible uses in the cosmetics and health industry, as well as to determine the efficacy of such products.

\section{Acknowledgements}

We express our sincerest gratitude to all those responsible for the maintenance and recording of the experimental flock at the Oudtshoorn Research Farm. We are grateful to the Klein Karoo Co-operation for financial and technical support and utilization of the South African Black resource flock, as well as to the commercial producer for entrusting his Zimbabwean Blue Neck breeding stock to us. Acknowledgment is given to the Technology and Human Resources for Industry Programme (THRIP) of the South African Department of Trade and Industry, the National Research Foundation (NRF) and the Food Security Programme of the HOPE Project of the University of Stellenbosch for funding this project.

\section{References}

Boylan, W.J., Berger, Y.M. \& Allen, C.E., 1976. Fatty acid composition of Finnsheep crossbred lamb carcasses. J. Anim. Sci. 42, 1421-1427.

Brand, M.M., 2006. Reproduction criteria and meat quality of South African Black (Struthio camelus var. domesticus), Zimbabwean Blue (Struthio camelus australis) and South African Black $\times$ Zimbabwean Blue ostriches. M.Sc. (Food Sci.) thesis, University of Stellenbosch, South Africa.

Department of Health, 1994. Nutritional aspects of cardiovascular disease. In: Report on Health and Social Subjects No. 46. London: Her Majesty's Stationery Office, UK.

Engelbrecht, A., Cloete, S.W.P. \& Van Wyk, J.B., 2008. Direct heterosis for liveweight and chick mortality in ostriches. Aust. J. Exp. Agric. 48, 1320-1325.

Fezler, D., 1995. Rhea oil: The most versatile and useful product of the Rhea americana. North American Rhea Association, March/April issue, 10-16.

Folch, J., Lees, M. \& Stanley, G.H.S., 1957. A simple method for the isolation and purification of total lipids from animal tissues. J. Biol. Chem. 226, 497-509.

Girolami, A., Marsico, I., D’Andrea, G., Braghieri, A., Napolitano, F. \& Cifuni, G.F., 2003. Fatty acid profile, cholesterol content and tenderness of ostrich meat as influenced by age at slaughter and muscle type. Meat Sci. 64, 309-315.

Glass, G.V., Peckham, P.D. \& Sanders, J.R., 1972. Consequences of failure to meet assumptions underlying the fixed effects analyses of variance and covariance. Rev. Edu. Res. 42, 237-288.

Gunstone, F.D. \& Russell, W.C., 1954. Animal fats. 3. The component acids of ostrich fat. Biochem. J. 57, 459-461.

Hoffman, L.C. \& Fisher, P., 2001. Comparison of meat quality characteristics between young and old ostriches. Meat Sci. 59, 335-337.

Hoffman, L.C., Joubert, M., Brand, T.S. \& Manley, M., 2005. The effect of dietary fish oil rich in n 3 fatty acids on the organoleptic, fatty acid and physicochemical characteristics of ostrich meat. Meat Sci. 70, 45-53.

Hoffman, L.C., Brand, M.M., Muller, M. \& Cloete, S.W.P., 2007. Carcass and muscle yields of ostriches as influenced by genotype. S. Afr. J. Anim. Sci. 37, 256-260. 
Hoffman, L.C., Muller, M., Cloete, S.W.P. \& Brand, M., 2008. Physical and sensory meat quality of South African Black ostriches (Struthio camelus var. domesticus), Zimbabwean Blue ostriches (Struthio camelus australis) and their hybrid. Meat Sci. 79, 365-374.

Horbaňczuk, J., Sales, J., Celeda, T., Konecka, A., Zieba, G. \& Kawka, P., 1998. Cholesterol content and fatty acid composition of ostrich meat as influenced by subspecies. Meat Sci. 50, 385-388.

Horbaňczuk, J.O., Malecki., I., Cooper, R.G., Jóźwik, A., Klewiec, J., Krzyżewski, J., Khalifa, H., Chyliński, W., Wójcik, A. \& Kawka, M., 2004. Cholesterol content and fatty acid composition of two fat depots from slaughter ostriches (Struthio camelus) aged 14 months. Anim. Sci. Pap. Rep. 22, 247-251.

Joubert, M., 2003. The manipulation of ostrich meat quality, composition and shelf life. MSc in Food Science thesis, University of Stellenbosch, South Africa.

Kemp, J.D., Mahyuddin, M., Ely, D.G., Fox, J.D. \& Moody, W.G., 1981. Effect of feeding systems, slaughter weight and sex on organoleptic properties and fatty acid composition of lambs. J. Anim. Sci. 51, 321-336.

Lanza, M., Fasone, V., Galofaro, V., Barbagallo, D., Bella, M. \& Pennisi, P., 2004. Citrus pulp as an ingredient in ostrich diet: effects on meat quality. Meat Sci. 68, 269-275.

Lawrie, R.A., 1998. Lawrie's Meat Science, $6^{\text {th }}$ ed. Woodhead Publishing Limited, Cambridge, England.

Mattson, F.H. \& Grundy, S.M., 1985. Comparison of effects of saturated, monounsaturated and polyunsaturated fatty acids on plasma lipids and lipoproteins in man. J. Lipid Res. 26, 194-202.

Mellett, F.D., 1992. Die volstruis as slagdier: Aspekte van groei. PhD in Agriculture thesis, University of Stellenbosch, South Africa.

Ott, R.L., 1998. An Introduction to Statistical Methods and Data Analysis. Belmont, California: Duxbury Press. pp. 807-837.

Paleari, M.A., Camisasca, S., Beretta, G., Renon, P., Corisco, P., Bertolo, G. \& Crivelli, G., 1998. Ostrich meat: physico-chemical characteristics and comparison with turkey and bovine meat. Meat Sci. 3, 205-210.

Sales, J., 1998. Fatty acid composition and cholesterol content of different ostrich muscles. Meat Sci. 49, 489-492.

Sales, J., 1999. Slaughter and products. In: The Ostrich, Biology, Production and Health. Ed. Deeming, D.C., Wallingford, Oxon: CABI Publishing. pp. 231-274.

Sales, J., Marais, D. \& Kruger, M., 1996. Fat content, caloric value, cholesterol content, and fatty acid composition of raw and cooked ostrich meat. J. Food Comp. An. 9, 85-89.

Sañudo, C., Nute, G.R., Campo, M.M., María, G., Baker, A., Sierra, I., Enser, M.E. \& Wood, J.D., 1998. Influence of weaning on carcass quality, fatty acid composition and meat quality in intensive lamb production systems. J. Anim. Sci. 66, 175-187.

SAS, 1999. SAS/STAT User's Guide, Version 9, $1^{\text {st }}$ printing, Volume 2. SAS Institute Inc, SAS Campus Drive, Cary, North Carolina 27513, USA.

Shapiro, S.S. \& Wilk, M.B., 1965. An analysis of variance test for normality (complete samples). Biometrika 52, 591-611.

Sinclair, A.J., Slattery, W.J. \& O’Dea, K., 1982. The analysis of polyunsaturated fatty acids in meat by capillary gas liquid chromatography. J. Food. Sci. Agric. 33, 771-776.

Stipanuk, M.H., 2000. Biochemical and Physiological Aspects of Human Nutrition. Pennsylvania, Philadelphia: W.B. Saunders Company, USA.

Tichelaar, H.Y., Smuts, C.M., Van Stuiivenberg, M.E., Faber, M. \& Benadé, A.J.S., 1998. The fatty acid composition of rural school children with helminth infections. Asia Pac. J. Clin. Nutr. 196-197.

Wang, Y.W., Sunwoo, H. \& Sim, J.S., 2000. Lipid characteristics of emu meat and tissue. J. Food Lipids 7, 71-82.

Whitehouse, M.W., Turner, A.G., Davis, C.K.C. \& Roberts, M.S., 1998. Emu oil(s): a source non-toxic transdermal anti-inflammatory agents in aboriginal medicine. Inflamm. 6, 1-7. 